\title{
Malignant peripheral nerve-sheath tumors in an adolescent patient with mosaic localized NF1: A case report
}

\author{
HIROKI HAGIZAWA $^{1 *}$, SHIGENORI NAGATA ${ }^{2 *}$, TORU WAKAMATSU $^{1}$, YOSHINORI IMURA $^{1}$, \\ TAKAAKI TANAKA ${ }^{1}$, HIDETATSU OUTANI ${ }^{3}$, EIICHI KONISHI ${ }^{4}$, NORIFUMI NAKA ${ }^{1}$ and HIRONARI TAMIYA ${ }^{1}$ \\ Departments of ${ }^{1}$ Orthopedic Surgery, and ${ }^{2}$ Diagnostic Pathology and Cytology, Osaka International Cancer Institute, \\ Osaka 541-8567; ${ }^{3}$ Department of Orthopedic Surgery, Osaka University Hospital, Suita, Osaka 565-0871; \\ ${ }^{4}$ Department of Pathology, Kyoto Prefectural University of Medicine, Kyoto 602-8566, Japan
}

Received June 10, 2019; Accepted October 8, 2019

DOI: $10.3892 / \mathrm{mco} .2019 .1969$

\begin{abstract}
Malignant peripheral nerve-sheath tumors (MPNSTs) are rare malignancies that are often observed in patients with neurofibromatosis type 1 (NF1). However, the occurrence of MPNST associated with mosaic localized NF1 is extremely rare. Previous reports have revealed that MPNST was associated with mosaic localized NF1 in only three patients who were $>40$ years of age. The present report details a 16 -year-old man who presented with pain and a $3 \mathrm{~cm}$ mass on the medial side of the right knee. Magnetic resonance imaging revealed a circumscribed soft tissue tumor located in the subcutaneous tissue. His previous doctor believed that it was benign and conducted a marginal resection. However, postoperative histology results demonstrated spindle cell sarcoma, following which the patient was referred to The Osaka International Cancer Institute. Localized café-au-lait spots were identified in the affected leg, which inferred that the patient had NF1-related MPNST. A wide resection was performed to completely resect the residual tumor; however, a definitive histological diagnosis was challenging due to the
\end{abstract}

Correspondence to: Dr Hironari Tamiya, Department of Orthopedic Surgery, Osaka International Cancer Institute, 3-1-69 Otemae, Chuo-ku, Osaka 541-8567, Japan

E-mail: tamiya-hi@mc.pref.osaka.jp

*Contributed equally

Abbreviations: NF1, neurofibromatosis type 1; MPNST, malignant peripheral nerve-sheath tumor; SOX10, SRY-related HMG-box 10; H3K27me3, trimethylation of lysine 27 of histone H3; SMA, smooth muscle actin; MDM2, mouse double minute 2; CDK4, cyclin-dependent kinase 4; CD, cluster of differentiation; CK, cytokeratin; BCL2, B-cell lymphoma 2; TLE1, transducin-like enhancer of split-1; STAT6, signal transducer and activator of transcription 6; PAX5, paired box 5; ER, estrogen receptor; PgR, progesterone receptor; RT-PCR, reverse transcriptase-polymerase chain reaction; PGP 9.5, protein gene product 9.5

Key words: neurofibromatosis type 1, mosaic localized, café-au-lait spot, malignant peripheral nerve-sheath tumor, adolescent small residual tumor. Hence, the genomic mutations of NF1 in the regional café-au-lait spots were analyzed. The result revealed an NF1 microdeletion and a consistently limited expression of NF1 in the tumor sample. Finally, the patient was diagnosed with MPNST with mosaic localized NF1. Local recurrence and distant metastasis were not observed 1.5 years after surgery. In conclusion, the present report presented MPNST in an adolescent patient with mosaic localized NF1. The occurrence of MPNSTs correlated with mosaic localized NF1 is extremely rare. However, it is of high-grade malignancy and therefore, its clinical features should be considered by orthopedists and pathologists.

\section{Introduction}

Neurofibromatosis type 1 (NF1) is also called von Recklinghausen disease, which is an autosomal dominant disorder characterized by café-au-lait spots, axillary and/or inguinal freckles, lisch nodules, and neurofibromas (1). Patients with NF1 occasionally present with malignant tumors arising from neurofibromas, which are referred to as malignant peripheral nerve-sheath tumors (MPNSTs), and the treatment for such a condition is challenging. The 5-year overall survival rate was approximately $34-64 \%$ in several studies. The mean age at diagnosis is 34.0 years, and patients with NF1 are younger than those without NF1 (mean age: 28.7 vs. 39.7 years) $(2,3)$.

Neurofibromatosis is classified into several subtypes. One subtype is the mosaic localized NF1 (also called NF5 or segmental NF), which is characterized by the local appearance of neurofibromatosis (4-6). Although most MPNSTs occur in patients with NF1, only three patients presented with mosaic localized NF1 $(7,8)$. The prevalence rate of mosaic localized $\mathrm{NF} 1$ is approximately $0.0018 \%$, whereas that of NF1 ranges from 0.02 to $0.03 \%(9,10)$. Such a result indicates the rarity of mosaic localized NF1.

Herein, we first report the case of MPNST in an adolescent patient with mosaic localized NF1. Initially, the patient underwent unplanned excision due to misdiagnosis with a benign tumor. The postoperative histologic diagnosis was spindle cell sarcoma; thus, a wide resection was conducted. The presence of segmental café-au-lait spots and freckles in the unilateral leg was helpful in diagnosing MPNST associated 
with mosaic localized NF1. Furthermore, NF1 microdeletion was confirmed in the café-au-lait spot, which was consistent with previous reports. Sparse NF1 expression was also observed in the tumor. Finally, the patient was diagnosed with MPNST with mosaic localized NF1.

\section{Case report}

A 16-year-old man presented with pain and a mass on the medial side of his right knee for 1.5 years prior to when he visited his first doctor. The tumor was approximately $3 \mathrm{~cm}$ in diameter. He underwent magnetic resonance imaging (MRI) that revealed a circumscribed soft tissue tumor located in the subcutaneous tissue. The tumor had an iso-signal intensity on T1-weighted sequences and high signal intensity on T2-weighted sequences (Fig. 1). The doctor diagnosed the tumor as benign and then performed a marginal resection. However, postoperative histological diagnosis revealed spindle cell sarcoma. The patient was then referred to our hospital for a more specialized treatment. We identified dense freckles and café-au-lait spots on his right inguinal region to the knee (Fig. 2). He had no family history of NF1. The segmental freckles led us to suspect MPNST associated with mosaic localized NF1. Preoperative MRI before additional wide resection showed that a residual tumor appeared to exist adjacent to the patella (Fig. 3A and B). We then confirmed the absence of metastasis and conducted an additional wide resection where part of the vastus medialis, the medial patellar retinaculum and joint capsule, and the patella were resected with the residual tumor. Soft tissue defect was reconstructed with pedicled anterolateral thigh musculocutaneous flap. There was no evidence of residual tumor and surgical margin proved to be negative (R0 resection) (Fig. 3C). The patient did not present with local recurrence and distant metastasis 1.5 years after surgery.

The pathologic review of the primary tumor was carried out with additional immunostainings at our institute (Vimentin, S100, SOX10, CD68, Ki67,H3K27me3, and NF1). Hematoxylin and eosin staining of the marginally removed mass was well-circumscribed and embedded in the subcutis showed spindle cell proliferation arranged in interlacing fascicules against a chronic inflammatory background, which comprised cellular areas alternating with less cellular areas, accompanied by hemangiopericytoma-like vessels and a small amount of extracellular myxoid matrix (Fig. 4A). Intratumoral necrosis was not apparent. The tumor cells had moderate-to-severe nuclear atypia with mitotic figures (count: 15/10 high-power fields) and focal pleomorphism (Fig. 4B). Unequivocal lipoblasts were not identified. An immunohistochemical study showed reactivity with variable cell populations and intensity to the following markers: Vimentin (Fig. 4C), desmin, aSMA, S100 (Fig. 4D), MDM2, CDK4, factor XIIIa, CD99, a1-antitrypsin, and a Ki67 labeling index of 20\% (Fig. 4E); the tumor cells were diffusely positive for vimentin in contrast to the form of scattered positive cells $(<5 \%)$ for S100. The negative markers and in situ hybridization (ISH) were as follows: Cytokeratins (AE1/AE3, CAM5.2, CK7, and CK19), caldesmon, myogenin, neurofilament, protein melan-A, CD21, CD23, CD30, CD31, CD34, CD68 (Fig. 4F), CD117, BCL2, TLE1, INI1, SOX10 (Fig. 4G), STAT6, PAX5, ER, PgR, and
Epstein-Barr encoding region ISH. The tumor partially lost the expression of H3K27me3 (Fig. 4H), whereas only a few neoplastic cells were positive for NF1 (Sigma-Aldrich; Merck $\mathrm{KGaA}$ ) (Fig. 4I). Immunostaining protocol is as follows; sections were hydrated by passage through xylene and graded ethanols. After antigen retrieval for $10 \mathrm{~min}$ at 99 degree in citric buffer, $\mathrm{pH}$ 6.0, the slides were blocked with $3 \%$ BSA for $1 \mathrm{~h}$, then incubated with a primary antibody for $16 \mathrm{~h}$ at 4 degree. After washing with PBS, slides were mounted using ImmPRESS HRP polymer detection kit (Vector Laboratories) and peroxidase Stain DAB kit (Brown Stain) (Nacalai Tesque), followed by counterstaining with hematoxylin.

With regards to genomic mutation, reverse transcriptase-polymerase chain reaction (RT-PCR) using the formalin-fixed and paraffin-embedded tissues of the primary tumor failed to detect SYT-SSX1 and SYT-SSX2 chimeric transcripts. NF1 microdeletion in the affected skin (Café-au-lait spot) was identified via RT-PCR using PrimeSTAR HS DNA polymerase (Takara Bio Inc.) although no mutation was observed in the healthy side. RNA was obtained using the RNeasy mini kit (Qiagen) after tissue homogenization and the extracted RNA was then retrotranscripted using SuperScript IV VILO Master Mix (Thermo Fisher Scientific, Inc.). As a DNA size marker, $1 \mathrm{~kb}$ or $100 \mathrm{bp}$ DNA Ladder (Takara Bio Inc.) was used. The primers used for amplifying a part of the NF1 gene were as follows: Exon 7-12: Forward 5'-AGATAA CTCTGTCATTTTCCTAC-3' and reverse 5'-CTATCCATA GAGGAGTTCGCT-3' as well as exon 36-46: Forward 5'-CCA GTGGACAGAACTA-GCTC-3' and reverse 5'-GGCCTC TGCTAAGTATTCATA-3'. As an internal control, GAPDH was measured using the following primers: Forward 5'-AGG GCTGCTTTTAACTCTGGT-3' and reverse 5'-CCCCACTTG ATTTTGGAGGGA-3' (Fig. 5).

\section{Discussion}

The etiology of NF1 is the mutation in the NF1 gene located in chromosome 17q11.2 (11). In 2000, Tinschert et al showed that mosaic localized NF1 was caused by the somatic mutation of the NF1 gene (12).

In 1977, Miller and Sparkes reported the case of a 15-year-old girl with multiple pigmented macules, café-au-lait spots, and a neurofibroma in a specific region of the body, which led to the establishment of the term segmental neurofibromatosis (4). In 1982, Riccardi proposed the classification of NF into eight subtypes. NF5 is segmental NF, which is defined by the limitation of café-au-lait spots, freckles, and/or cutaneous neurofibromas to a certain region of the body (5). In 1987, Roth et al further classified the segmental NF into four categories: True segmental (NF5), localized with deep involvement, hereditary segmental, and bilateral segmental (13). In 1997, Gutmann et al proposed the term mosaic NF1 (14). In 2001, Ruggieri and Huson further classified mosaic NF1 into two categories: Mosaic generalized NF1 and mosaic localized NF1 (6). Based on pathogenesis, these terms represent varying skin lesions, affected regions, and symptoms accurately.

The prevalence rate of mosaic localized NF1 is approximately $0.0018 \%$, whereas that of NF1 is approximately $0.02-0.03 \%(9,10)$. Meanwhile, the incidence rate of MPNST is $4.6 \%$ in individuals with NF1 and $0.001 \%$ in the general 
A

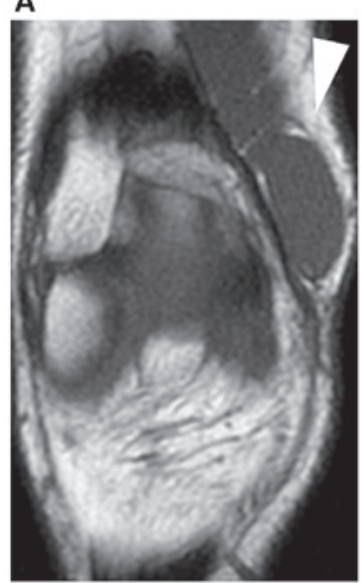

B

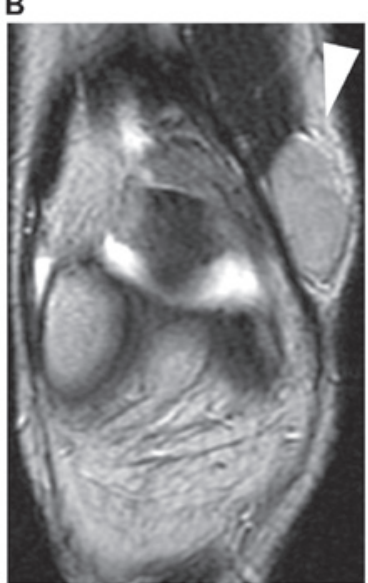

C

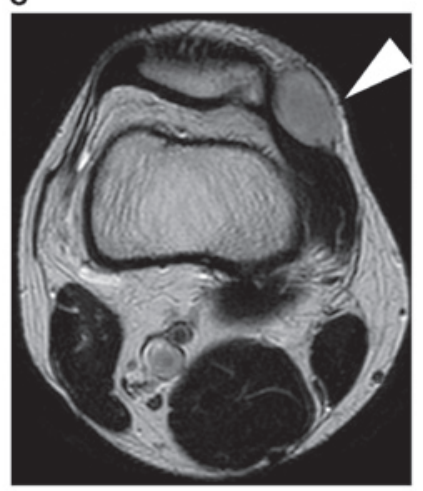

Figure 1. Coronal and axial images obtained during the first round of preoperative magnetic resonance imaging. (A) coronal T1-weighted, (B) coronal T2-weighted and (C) axial T2-weighted images are presented. White arrowheads indicate the tumor.

A

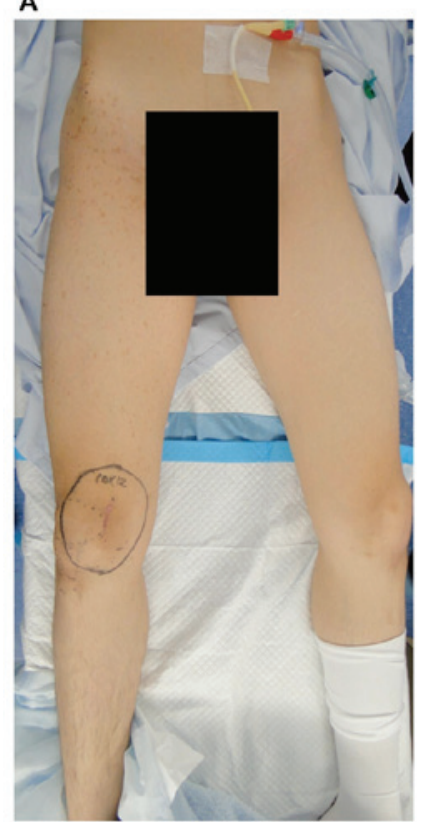

B

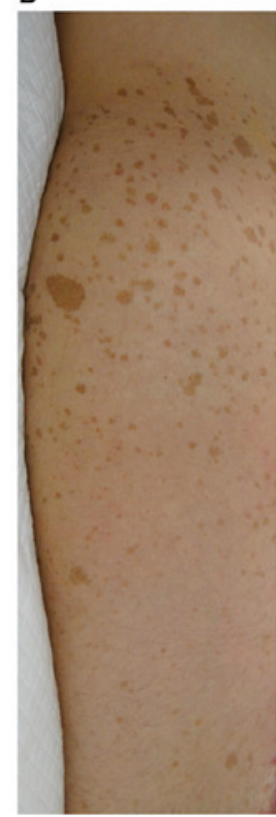

Figure 2. Dense freckles are presented on the patients' right inguinal region to the knee, as observed during surgery. Segmental freckles were limited to (A) the unilateral side and (B) localized café-au-lait spots were identified.

population, and approximately $52 \%$ of patients with NF1 presented with MPNST (2). Although numerous patients with NF1 present with MPNST, only three cases involved mosaic localized NF1 $(7,8)$. There are two hypotheses for this phenomenon. First, cells with gene mutation in mosaic localized NF1 are limited to the affected region and the number of cells that can be mutate to MPNST is higher in NF1 than in mosaic localized NF1 (7). Second, mosaic localized NF1 is underdiagnosed (9). In addition, we hypothesized that the proportion of patients with mosaic localized NF1 who present with neurofibroma is low, although MPNST arises in the neurofibroma of patients with NF1 in which malignant transformation occurs. Ruggieri et al classified 124 patients with mosaic localized NF1 into four groups: Pigmentary changes only, neurofibromas only, pigmentary changes and neurofibromas, and isolated plexiform neurofibromas only. A total of 86 patients were included in the pigmentary change only group, 20 in the neurofibroma only group, 10 in the pigmentary change and neurofibroma group, and 8 in the isolated plexiform neurofibroma only group. Pigmentary changes were often observed (6).

The resection of the tumor is the definitive treatment for MPNST and the efficacy of adjuvant or neoadjuvant therapy has not been fully investigated. The local recurrence rates of resection with adequate surgical margin and with inadequate surgical margin were 6 and $30 \%$, respectively $(2,3)$.

An earlier clinical report showed that the 10 -year overall survival rate of individuals with NF1-related MPNST and sporadic MPNST are 45 and 60\%, respectively, and statistically significant differences were observed (15). The number of individuals with mosaic localized NF1 is extremely low; thus, studies about the survival rate of patients with MPNST arising from mosaic localized NF1 were not conducted. In a case report, all three cases survived at least during the follow-up period although two cases out of three caused local recurrence (8). In the present case, we identified NF1 microdeletion; thus, a cautious follow-up is required not to overlook local recurrence and new lesions in the affected leg.

The features of the histopathological findings of MPNST are spindle cell tumors growing in storiform and positive in immunohistochemical staining, which include S-100, CD56, and protein gene product 9.5 (PGP 9.5). In particular, S-100 was considered a good marker of MPNST, but it is only about $50-90 \%$ positive (16) and there is no immunohistochemical marker with high sensitivity and specificity for MPNST. Previous studies have also reported on patients diagnosed with spindle cell sarcoma associated with mosaic localized NF1 and S-100 positive as MPNST $(7,8)$. Synovial sarcoma was excluded because neither SYT-SSX1 nor SYT-SSX2 fusion gene was identified via RT-PCR. Other types of sarcomas, such as dedifferentiated liposarcoma, leiomyosarcoma, rhabdomyosarcoma, or solitary fibrous tumor, are significantly less common in younger patients, and their morphological and immunohistochemical findings were inconsistent. Finally, MPNST was the most probable diagnosis based on the immunohistochemical analysis. 
A

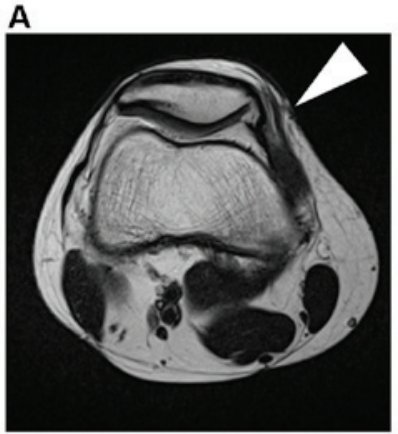

B

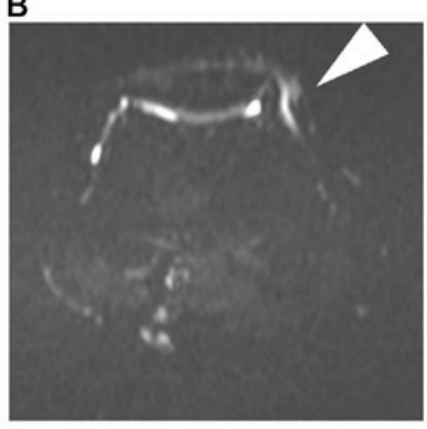

C

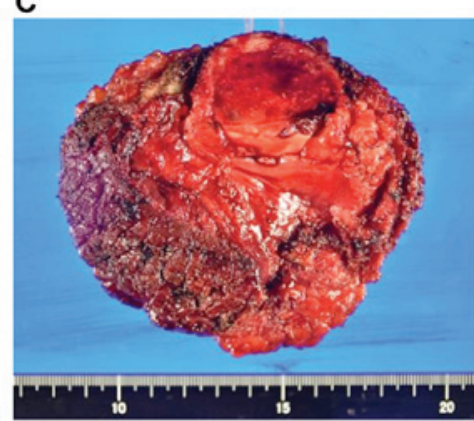

Figure 3. Preoperative images obtained prior to the additional wide resection and the resected specimen. (A) Axial T2-weighted and (B) diffusion-weighted images (B) are presented. (C) The resected specimen is also presented. White arrowheads indicate residual tumor and/or postoperative inflammatory responses.

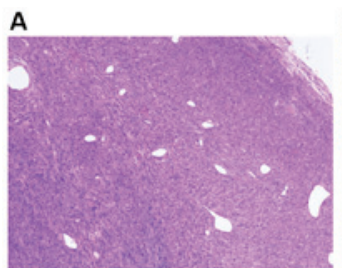

D

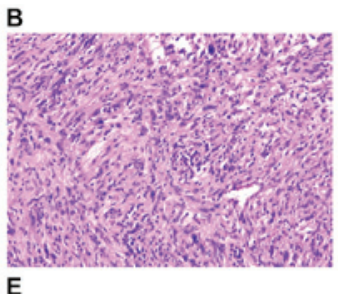

C

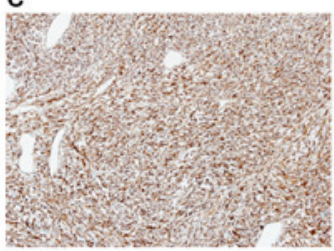

$\mathbf{F}$

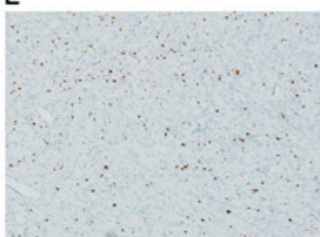

H

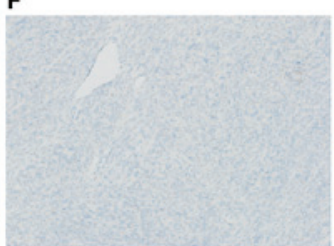

I

G

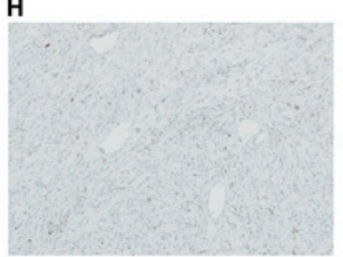

Figure 4. Microphotographs of the surgically removed tumor. (A) A well-circumscribed mass presenting dense spindle-cell proliferation with hemangiopericytomatous vessels was observed (H\&E staining; magnification, x40). (B) Neoplastic cells exhibiting moderate-to-severe nuclear atypia and focal pleomorphism against a chronic inflammatory background (H\&E staining; magnification, x200). (C) Diffuse positivity for vimentin (IHC staining; magnification, x100) was observed. (D) Scattered positive cells $(<1 \%)$ for S100 (IHC staining; magnification, x100) were identified. (E) A Ki67 labeling index of $20 \%$ was determined (IHC staining; magnification, x100). Samples were negative for (F) SOX10 (IHC staining; magnification, x100) and (G) CD68 (IHC staining; magnification, x100). Incomplete loss of expression of (H) H3K27me3 (IHC staining; magnification, x100) and (I) NF1 (IHC staining; magnification, x100). H\&E, hematoxylin and eosin; IHC, immunohistocemical.

A

\begin{tabular}{|c|c|c|c|c|}
\hline \multirow[t]{2}{*}{ Exon } & \multicolumn{2}{|c|}{$7-12$} & \multicolumn{2}{|c|}{$36-46$} \\
\hline & 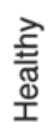 & $\begin{array}{l}\text { d } \\
\frac{d}{J} \\
\frac{\mathbb{J}}{4}\end{array}$ & $\begin{array}{l}\text { } \\
\text { 志 } \\
\text { I্ }\end{array}$ & $\begin{array}{l}\text { ర్త } \\
\frac{\mathbb{d}}{4} \\
\frac{4}{4}\end{array}$ \\
\hline
\end{tabular}

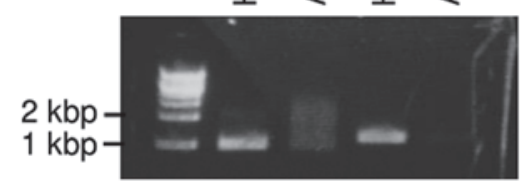

B

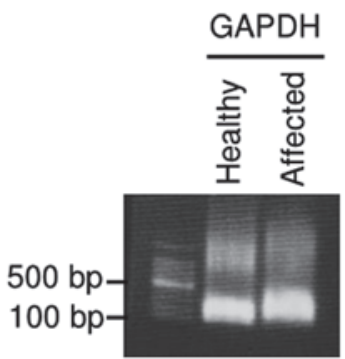

Figure 5. NF1 gene expression in healthy and affected skin. (A) Exon amplification in NF1 was observed, with (B) GAPDH serving as an internal control.

The definitive diagnosis of MPNST was challenging in the present case. A previous report has revealed the diagnosis of
MPNST and showed that all MPNSTs are characterized by NF1 deletion, although NF1 expression varies in plexiform neurofibroma, indicating that the deletion of NF1 is a useful indicator of appropriate diagnosis (17). In addition, NF1 microdeletion was observed in both generalized and localized NF1 (18). Thus, our patient can be diagnosed with MPNST arising from mosaic localized NF1 due to the presence of regional café-au-lait spots and genomic NF1 deletion.

Neurofibromatosis type 1 results from NF1 microdeletion which encompasses the entire NF1 gene. In Fig. 5, not only exon 36-46, but exon 7-12 was also deleted in the affected skin. This huge deletion of NF1 means NF1 microdeletion, which is observed only in neurofibromatosis type 1. As far as we checked on the database of COSMIC, NF1 microdeletion is not observed in any kinds of cancer other than neurofibromatosis type 1. NF1 microdeletion is an absolute causative gene (100\% penetrance), not a second hit. 
Earlier reports identified three patients aged $>40$ years who presented with MPNST associated with mosaic localized NF1. We reported the first case of MPNST in an adolescent patient with mosaic localized NF1. Moreover, malignant transformation in neurofibroma was assumed to occur during puberty due to the fact that the exacerbation of neurofibroma during puberty and pregnancy is well recognized (19).

In this report, MPNST was observed in an adolescent patient with mosaic localized NF1. In the case of a challenging histologic diagnosis, the presence of skin lesions is helpful in the diagnosis of MPNST. In the present study, NF1 microdeletion was consistently observed in the café-au-lait spots. MPNST associated with mosaic localized NF1 is a rare type of malignancy. However, it is of high-grade malignancy; therefore, orthopedists should bear in mind this clinical feature.

\section{Acknowledgements}

Not applicable.

\section{Funding}

No funding was received.

\section{Availability of data and materials}

The datasets used and/or analyzed during the present study are available from the corresponding author on reasonable request.

\section{Authors' contributions}

$\mathrm{HH}, \mathrm{SN}$, and HT conceived the present study, pathologically diagnosed the patient and wrote the manuscript. SN, EK, and HT performed cytologic diagnoses, immunocytochemical analysis and gene expression analysis. HH, TW, YI, TT, HO, NN and HT collected clinical data. SN and HT revised the manuscript. All authors read and approved the final manuscript prior to submission.

\section{Ethics approval and consent to participate}

Skin samples were collected after written informed consent was obtained according to the protocol approved by Osaka International Cancer Institute (Osaka, Japan). Written informed consent was obtained from the patient and patient's parents for study participation.

\section{Patient consent for publication}

Written informed consent was obtained from the patient and patient's parents for the publication of patient data and associated images.

\section{Competing interests}

The authors declare that they have no competing interests.

\section{References}

1. Fletcher CDM, Unni KK and Mertens F: Pathology and genetics of tumours of soft tissue and bone. WHO classification of tumours. IARC Press Lyon, 2002.

2. Ducatman BS, Scheithauer BW, Piepgras DG, Reiman HM and Ilstrup DM: Malignant peripheral nerve sheath tumors. A clinicopathologic study of 120 cases. Cancer 57: 2006-2021, 1986.

3. Porter DE, Prasad V, Foster L, Dall GF, Birch R and Grimer RJ: Survival in malignant peripheral nerve sheath tumours: A comparison between sporadic and neurofibromatosis type 1-associated tumours. Sarcoma 2009: 756395, 2009.

4. Miller RM and Sparkes RS: Segmental neurofibromatosis. Arch Dermatol 113: 837-838, 1977.

5. Riccardi VM: Neurofibromatosis: Clinical heterogeneity. Curr Probl Cancer 7: 1-34, 1982.

6. Ruggieri M and Huson SM: The clinical and diagnostic implications of mosaicism in the neurofibromatoses. Neurology 56: 1433-1443, 2001.

7. Schwarz J and Belzberg AJ: Malignant peripheral nerve sheath tumors in the setting of segmental neurofibromatosis. Case report. J Neurosurg 92: 342-346, 2000.

8. Li K, Chong HW and Sang EM: A superficial form of malignant peripheral nerve sheath tumour associated with segmental neurofibromatosis. Acta Derm Venereol 85: 540-541, 2005.

9. Ingordo V, D'Andria G, Mendicini S, Grecucci M and Baglivo A: Segmental neurofibromatosis: Is it uncommon or underdiagnosed? Arch Dermatol 131: 959-960, 1995.

10. Lammert M, Friedman JM, Kluwe L and Mautner VF: Prevalence of neurofibromatosis 1 in German children at elementary school enrollment. Arch Dermatol 141: 71-74, 2005.

11. Ledbetter DH, Rich DC, O'Connell P, Leppert M and Carey JC: Precise localization of NF1 to 17q11.2 by balanced translocation. Am J Hum Genet 44: 20-24, 1989.

12. Tinschert S, Naumann I, Stegmann E, Buske A, Kaufmann D, Thiel G and Jenne DE: Segmental neurofibromatosis is caused by somatic mutation of the neurofibromatosis type 1 (NF1) gene. Eur J Hum Genet 8: 455-459, 2000.

13. Roth RR, Martines R and James WD: Segmental neurofibromatosis. Arch Dermatol 123: 917-920, 1987.

14. Gutmann DH, Aylsworth A, Carey JC, Korf B, Marks J, Pyeritz RE, Rubenstein A and Viskochil D: The diagnostic evaluation and multidisciplinary management of neurofibromatosis 1 and neurofibromatosis 2. JAMA 278: 51-57, 1997.

15. Hwang IK, Hahn SM, Kim HS, Kim SK, Kim HS, Shin KH, Suh CO, Lyu CJ and Han JW: Outcomes of treatment for malignant peripheral nerve sheath tumors: Different clinical features associated with neurofibromatosis type 1 . Cancer Res Treat 49: 717-726, 2017.

16. Stasik CJ and Tawfik O: Malignant peripheral nerve sheath tumor with rhabdomyosarcomatous differentiation (malignant triton tumor). Arch Pathol Lab Med 130: 1878-1881, 2006.

17. Perry A, Roth KA, Banerjee R, Fuller CE and Gutmann DH: NF1 deletions in S-100 protein-positive and negative cells of sporadic and neurofibromatosis 1 (NF1)-associated plexiform neurofibromas and malignant peripheral nerve sheath tumors. Am J Pathol 159: 57-61, 2001.

18. Messiaen L, Vogt J, Bengesser K, Fu C, Mikhail F, Serra E, Garcia-Linares C, Cooper DN, Lazaro C and Kehrer-Sawatzki H: Mosaic type-1 NF1 microdeletions as a cause of both generalized and segmental neurofibromatosis type-1 (NF1). Hum Mutat 32: 213-219, 2011.

19. Riccardi VM: Von Recklinghausen neurofibromatosis. N Engl J Med 305: 1617-1627, 1981.

This work is licensed under a Creative Commons Attribution-NonCommercial-NoDerivatives 4.0 International (CC BY-NC-ND 4.0) License. 